\title{
Bitcoins in literature Review
}

\author{
Ahmed Elsayed Hamdallah * \\ Hamdy Mahmoud Kadous ** \\ Rana Mahmoud Abdou ***
}

*Ahmed Elsayed Hamdallah: Professor of Financial Accounting faculty of commerce and business administration, Helwan university.

** Hamdy Mahmoud Kadous: Professor of Financial Accounting faculty of commerce and business administration, Helwan university.

*** Rana Mahmoud Abdou: Assistant lecturer, faculty of commerce and business administration, Helwan university.

Email : Alaa_serafy@yahoo.com 


\section{Abstract:}

In recent years, cryptocurrencies have been in the spotlight of attention in financial markets all over the world. bitcoin, which was the first currency of this technology to be invented, still remains the most popular digital currency; however, other alternatives such as Ethereum and Ripple are gaining pace.

Bitcoin, introduced in October 2008 by Nakamoto, is a decentralized cryptocurrency. It has received increasing attention by business companies, organizations and governments since it is launched.

Over the last decade that bitcoin has been traded, many alterations have taken place from exchanges to the likelihood of closure. Nevertheless, the bitcoin has unique default benefits and properties by its structure. It is fully decentralized and depends on a sophisticated cryptographic protocol that it is difficult to counterfeit.

In this research the researcher clarified the nature, characteristics , pros and cons, and also how the bitcoin system works, through reviewing number of papers, accounting academic journals , professional publications .

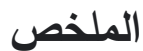

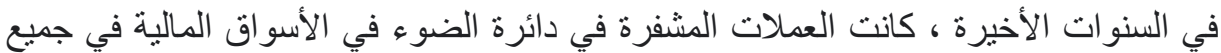

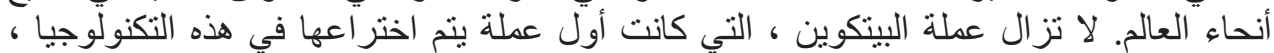

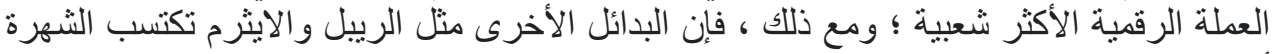

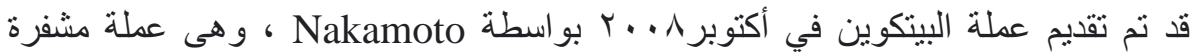
لامركزية. لقد حظيت باهتمام منزايد من قبل الثركات التجارية و المنظمات و الحكومات منذ

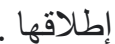

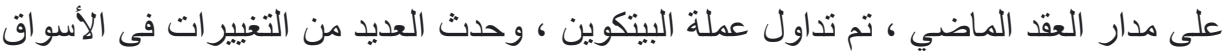

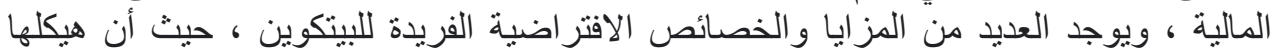

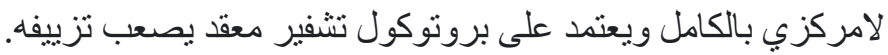

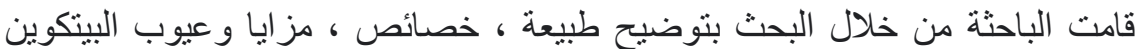

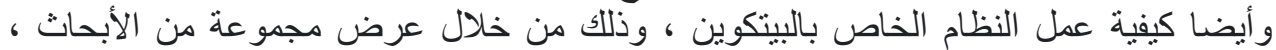
المجلات العلمية و المقالات المنينيه . 


\section{Introduction:}

There are many opinions about the role that bitcoin plays in the economy. It is viewed as an alternative to fiat currencies or even as part of an alternative economy. The role of bitcoin as a currency has been questioned by market experts, especially due to its volatility and the speed of transaction processing (Agata ,.et al, 2019, p.247 ) , However Bitcoin is by far the most valuable cryptocurrency with a capital market share of around $\$ 92$ billion as of April 2019 . (Ghada ,2019, P.34)

By a variety of metrics, bitcoin is no longer a financial curiosity. From its genesis transaction of 50 bitcoins in January 2009, bitcoins in circulation now number more than 17 million. An estimated 35 million Bitcoin wallets are held worldwide with $10 \quad 0,0 \quad 0 \quad 0$ companies accepting payments in bitcoins, some via the newly issued bitcoin debit card. ( David ,.et al, 2019, P. 91)

\section{Bitcoins Nature and Characteristics:}

\section{1 bitcoin The Currency :}

bitcoin is a special kind of assets called cryptocurrency. It was designed by Satoshi Nakamoto (allegedly a pseudonym of one person or a group of people) to work as a medium of exchange.( (Agata Kliber , Paweł Marszałek , Ida Musiałkowska, Katarzyna Świerczyńska, 2019, P.247) So it is a virtual monetary unit and therefore has no physical representation.

A bitcoin unit is divisible and can be divided into 100 million "Satoshis," the smallest fraction of a bitcoin. However, the currency unit used in payments on the Bitcoin network is bitcoins, not a fiat currency. Therefore, bitcoins in itself is also a digital currency, in the sense that it exists "digitally" and, for most intents and purposes, satisfies the economic definition of money: it is a medium of exchange, unit of account, and store of value. (Matonis, 2013, P.3).But contrary to "traditional" fiat currencies, bitcoin does not rely on any central authority, but uses cryptography to control its creation and management. (Agata Kliber, Paweł Marszałek , Ida Musiałkowska, Katarzyna Świerczyńska, 2019, P.247)

Transactions occur directly between clients and are logged into a central database known as a ledger. If a client chooses to use an 
anonymous server to conduct the transaction, then the owner of this transaction will be unidentified; however, the transaction will always be recorded in the open ledger. (Mohammed Ahmad Naheem , 2019 , P.517 ) .There are three ways to obtain bitcoins: exchanging money, selling goods and services or mining. The first two ways, which are exchanging money and selling goods or services through e-businesses that accept bitcoin units, make bitcoin behave like a fiat currency. (Elise Alfieri, Radu Burlacu and Geoffroy Enjolras,2019, P P.1114-115).

Bitcoin is currently a payment system that operates outside the realm of the banking sector, although once money has been converted into fiat (legal tender currencies) through money exchanges, then the banking sector can become involved. (Mohammed Ahmad Naheem , 2019 , P.517 ) However, a fundamental aspect that makes bitcoin behave like a financial asset is that one may create bitcoins through the mining process, whose underlying technology is named "Blockchain". This is a secured and distributed database that contains the history of transactions. The technology may be considered as a ledger that stores all exchanges realised on the network (Elise Alfieri, Radu Burlacu and Geoffroy Enjolras, 2019, P P.1114-115)

Bitcoin is basically a payment system that is independent from any government and operates without a third party (such as a Central Bank). Any participant in this system may check the behaviour of other participants to ensure the reliability of transactions and system stability. In other words, the wellfunctioning responsibility is not assumed by a third party. Moreover, all participants have the possibility to know the transactions made by everyone. (Elise Alfieri, Radu Burlacu and Geoffroy Enjolras,2019 , P P.114-115)

Conventionally, the uppercase "Bitcoin" refers to the network and technology, while the lowercase "bitcoin(s)" refers to units of the currency. The currency is also commonly abbreviated to "BTC," although some exchanges use "XBT," a proposed currency code that is compatible with ISO 4217 (Matonis, 2013, P.4).

\subsection{Bitcoin The Network :}

Bitcoin is programmed to record all transactions into a new block approximately every $10 \mathrm{~min}$. When a member of the network verifies the transactions of a block, and solves the mathematical 
Proof-of-Work to associated with it, they are rewarded with newly issued bitcoins . Essentially, Bitcoin is a technology for the conversion of electricity and processing power into accurate records, rewarding members to the extent they expend resources on verification. Network members expend processing power on verifying validity of transactions, and verifying each other's verification in a highly complex iterative process that requires vast quantities of processing power and electricity but produces a ledger of ownership and transactions that is beyond dispute, without having to rely on the trustworthiness of any single third-party. Bitcoin is built on $100 \%$ verification and $0 \%$ trust. (Saifedean Ammous , 2018 , P. 4)

\subsection{History of Bitcoin:}

"On Halloween of 2008, an entity named Satoshi Nakamoto distributed a white paper through metzdowd.com," (Darlington, 2014 , p.2). The term "entity" is used in relation to Satoshi Nakamoto, as the true identity of Satoshi is not known.

The paper titled bitcoin: A Peer to Peer Electronic Cash System described the system along with its underlying technology, the block chain database. The paper primarily addressed, and was the first to solve, the double spending problem associated with electronic currencies. The paper further discussed a peer-to peer system for transactions without the need for a third party trust agent. What the paper described however was a new trust system entirely, independent of third parties: the blockchain database. In January of 2009, the first reference implementation of Bitcoin was released by Nakamoto as an online monetary system (Baros, 2014, p. 211).

Nakamoto then created a website with the domain of bitcoin.org and Nakamoto continued to work with other developers on the code base through this site until around mid- 2010, according to bitcoin.org's 'About Us' page. At this time Nakamoto handed over control of the source code repository and network alert key to Gavin Andresen. Nakamoto also transferred several related domains to prominent contributors to the initial work. Since the mid 2010 date Andresen has acted as Bitcoins chief developer, and in 2012 the Bitcoin Foundation was formed. (Baros, 2014, p. 212). 
This organization has served as the Bitcoin community's public face since its creation. While bitcoin has seen large-scale adoption and increase in value since its inception in 2009, there have been several negative incidents along the way. The first of these setbacks occurred in August 2010. A major flaw in the blockchain ledger (transaction $\log$ ) was discovered, and allowed hackers to create an indefinite number of Bitcoins (Shubber, 2014, P.3). The hackers managed to create 184 million new bitcoins. This hack was quickly discovered and the blocks (transaction records) deleted in the database. The code base forked to a new code base that plugged the vulnerability. In 2014 a bug in the Bitcoin code was revealed that allowed malicious users to report frau dulent transactions that should not have been allowed to enter the ledger (Darlington, 2014, P.4).

This issue has since been fixed and the price of bitcoin continued to rise. While these bugs attacked at the periphery of the Bitcoin system, major security issues with the core of the code have not been prevalent. While technical problems have been found with the bitcoin wallet (Mainelli \& Smith, 2015) Overall faith in the technical underpinnings of the currency has continued. Bitcoin has the ability to change the underlying code and rules of currency. Increase in transactions caused problems for bitcoin to handle the pace (Titcomb, 2017, p. 2).

\section{$\underline{2.4}$ Is bitcoin "Digital" Gold or Cash?}

Some researchers consider the possibility that bitcoin acts like a commodity, more precisely as gold, for some reasons: supply is limited, meaning a weak inflation rate; monetary creation is based on the "mining" process; there is no control by any government; and bitcoin acts as a medium of exchange. Precious commodities such as gold are relatively safe, and bitcoin may often play a role of safe investment

For example, after the Cyprus crisis in 2012-2013, some depositors exchanged euros for bitcoins because of the bankruptcy of banks and also because of increased taxes on deposits. The second peak at US $\$ 1,000$ was linked to the international context in both developed and emerging countries. Chinese depositors turned toward bitcoin because of the Yuan drop and Chinese restrictions on capital outflows (this trend was accelerated by Donald Trump's election in the USA). 
Likewise, unstable monetary policies in emerging countries (inflation in Venezuela, demonetisation in India, liquidity crisis in Zimbabwe) encouraged local depositors to turn to bitcoin. Empirically, bitcoin appears to be a weak safe investment reserved to some special cases but not during financial crises . (Elise Alfieri, Radu Burlacu and Geoffroy Enjolras, 2019 , P P.117-118)

While bitcoin may act as a safe investment in some cases, we can observe major differences with precious commodities such as gold. First, bitcoin is capped at 21 million and the release of new bitcoins is divided by two approximately every four years until the maximum number of bitcoins is reached. However, the supply for gold is not known. Moreover, even if the price of bitcoin increases, its capitalization (US\$117,353,600,811 in November 2017) is much lower than for gold (US\$7,747,981,667,403 in November 2017) Second, the price of bitcoin is independent from that of gold.

The factors affecting the value of these two assets are different. Finally, gold (or any commodity) has physical shape, whilst bitcoin possesses a virtual one. An alternative complementary category is proposed by Selgin, a category named "Synthetic commodity money" that is both commodity money and fiat money . (Elise Alfieri, Radu Burlacu and Geoffroy Enjolras,2019, P P.117-118)

However gold is useful as an alternative to holding government backed or issued currency, it do not act as currency in today's large international markets. bitcoins or cryptocurrencies are not government backed so it derives value as an alternative to government backed currency. bitcoin holds an advantage over gold and other securities as it is more easily spent or exchanged in markets for nearly any commodity or service. (Kharpal, 2017, P.5).

Finally, bitcoin could also be assimilated to cash or to a cash equivalent. But a cash equivalent implies that the asset must be highly liquid and convertible into a known amount of cash. Bitcoin is convertible, but it is not enough liquid to be considered as cash equivalent. Bitcoin cannot be deposited into banks and cannot be withdrawn using ATM. (Elise Alfieri, Radu Burlacu and Geoffroy Enjolras,2019, P P.117-118)

\subsection{The System of Bitcoin :}

The technology principle underpinning Bitcoin is the transaction database called a blockchain. bitcoin is a mathematical currency, so transactions require an algorithmic process to occur, which is 
known as block building. The blockchain logs every transaction that has involved the bitcoin and adds a hash of the last transaction to the next block in the chain. So, there is a numerical and sequential record that cannot be altered very easily, without affecting all the other chains in the block. (Mohammed Ahmad Naheem , 2019 ,P.517 )

To a layperson, bitcoin is a digital currency that is created and held electronically. These bitcoins are sent and received using a mobile app, computer software, or service provider that provides a bitcoin wallet. The wallet generates an address, akin to a bank account number, except that a Bitcoin address is a unique alphanumeric sequence of characters where the user can start to receive payments. Usually, bitcoins may be obtained by buying them at a Bitcoin exchange or vending machine or as payment for goods and services.

However, Bitcoin is revolutionary because the double-spending problem can be solved without needing a third party. In computer science, the double-spending problem refers to the problem that digital money could be easily spent more than once, Bitcoin solves the double-spending problem by maintaining a ledger of balances, but instead of relying on a single trusted third party to manage this ledger, Bitcoin decentralizes this responsibility to the entire network. Behind the scenes, the Bitcoin network constantly keeps track of bitcoin balances in a public ledger called the blockchain.

The blockchain is a publicly accessible authoritative record of all transactions ever processed, allowing anyone to use Bitcoin software to verify the validity of a transaction. Transfers of bitcoins, or transactions, are broadcast to the entire network and are included onto the blockchain upon successful verification, so that spent bitcoins cannot be spent again. New transactions are checked against the blockchain to make sure that the bitcoins have not been already spent, thus solving the double-spending problem. (handbook of digital currency ,2015,p.39)

Bitcoin extensively uses public-key cryptography to solve the double-spending problem. In public-key cryptography, each transaction has a digital signature and contains a hash that allows for easy tamper detection merchants who accept bitcoins for both digital and physical goods and services. bitcoins are typically stored in a wallet, so a user needs to have a wallet available to buy 
and sell bitcoins. Specifically, it is the private keys that are stored in a wallet (CoinDesk, 2014). These keys are used to access the Bitcoin addresses and sign transactions and therefore must be kept securely (Bitcoin.org).

bitcoin miners are volunteers that work at solving the algorithm in return for payment; once a consensus is reached on the algorithm, then the block can be added to the chain. It is this system of verification and proof of work that provides the security of the currency, as it cannot be easily altered and leaves a permanent record of the transactions. It is also a value system that is not built on trust, unlike other value trading commodities such as the wine or art markets that can fluctuate due to trust issues. (Mohammed Ahmad Naheem , 2019 ,P.517 )

\section{How Does Bitcoin Work?}

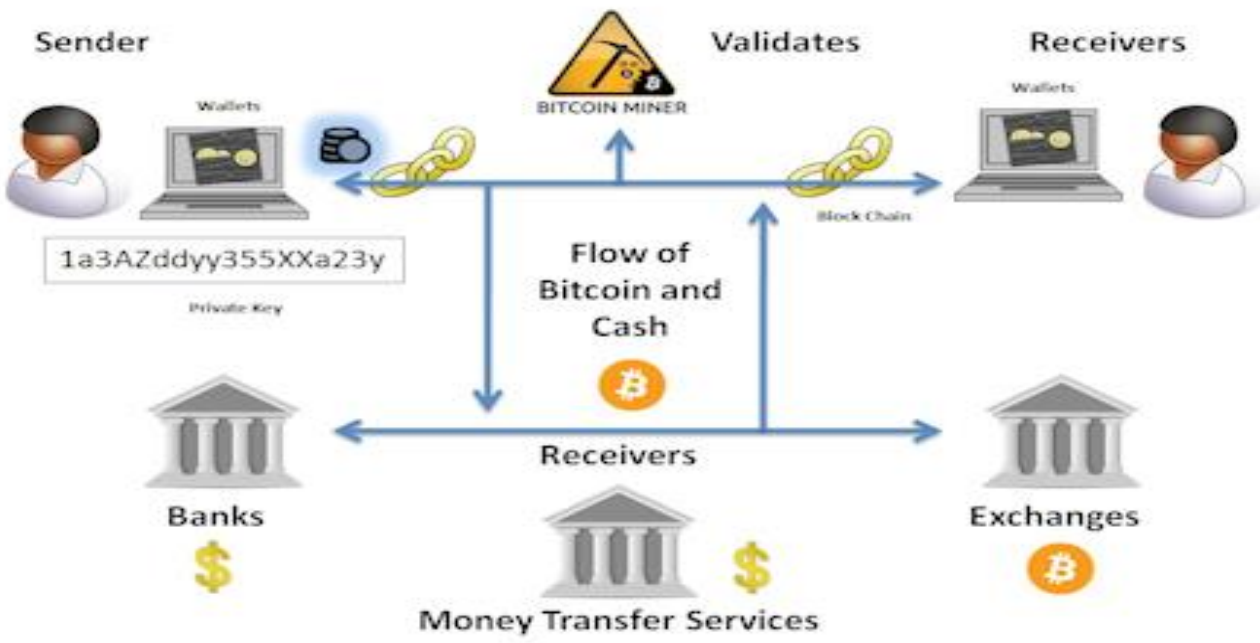

Image source : https://www.bitcoinsnitch.com

\subsection{Parties Involve In bitcoins Transactions : (CPA ,2017,PP.222-223)}

A sizable and vibrant cryptocurrency ecosystem has developed over time in several leading jurisdictions-including Singaporewith a number of prominent venture capital firms having invested, and continuing to invest, in various cryptocurrency start-ups and businesses. 
This ecosystem broadly comprises participants such as miners, users, exchangers, and transaction service providers and software developers, among many other stakeholders:

1. Miners : are persons or entities that run specialized software to generate solutions to complex algorithms and verify transactions in the cryptocurrency network.

2. Users: are persons or entities that obtain cryptocurrency and use it to purchase goods or services, or to transfer value to another person, or to hold for investment purposes.

3. Exchangers : are persons or entities in the business of exchanging cryptocurrencies for real or fiat currency, such as the US dollar or the Japanese yen, or for other cryptocurrencies or virtual currencies.

4. Transaction service providers : are websites that provide transaction services, allowing individuals to store and transact Bitcoins without having to run the Bitcoin client on their own computers. This includes wallet and vault providers.

5. Software developers : are persons or entities that are involved in researching, designing, making, or testing computer software that makes use of cryptocurrencies.

6. Other participants in the cryptocurrency ecosystem : include market information and chart providers and merchants that accept cryptocurrencies in exchange for real goods and real services.

2.7 Characteristics of Money Versus bitcoins : (Ehsan Nikbakht, Manuchehr Shahrokhi, Alford Corriette,2018 , P.P.5-7)

As it pertains to the true economic definition of money, can a valid argument be made in justifying Bitcoin (although not a physical coin) as a tool (asset) to be efficiently implemented in transactions?

To develop a plausible answer, we use the three parts/characteristics of money, better knowns as the "markers" of what money should be:

1. unit of account : bitcoins can be used in bookkeeping processes. As the blockchain technology focuses on ledgers which are records of transactions. These records are stored and shared to the consensus,.

2. Store of value : as related to bitcoin, it implies that specific quantities of bitcoin lead to a specific worth. Unlike bitcoin, fiat currency's value is dictated by a financial institution. 
3. medium of exchange: is particular when blockchain-supported currencies are investigated. With bitcoin, exchanges would most likely occur via computerized systems. As it is primarily digital, any transfer of the currency does not have a physical transfer as fiat currency does. In addition, it makes the currency vulnerable in the event of cyber breaches.

From an economic perspective, the question whether bitcoin has common characteristics with currency is subject to debate. According to its basic properties, a currency should be a convenient medium of exchange, a stable unit of account and a durable store of value .bitcoin is a medium of exchange, in the sense that a high number of businesses, such as Dell, Microsoft or PayPal, are willing to accept bitcoins (Figuet, 2016,P.326).

bitcoin may be considered to some extent as a "unit of account". However, merchants do not display prices in bitcoin for two reasons. First, its supply is inelastic (a bitcoin price of a given product needs many digits after the comma). Second, volatility does not ensure price stability. Because of high volatility, merchants are forced to change frequently the price of their products. So, prices are usually displayed in US dollars and are then converted in bitcoins at the time when the transaction takes place (Figuet, 2016, P.327).

Finally, while bitcoin has features that make it behave like a "store of value", the possible cybersecurity risks reduce trust in this currency. The trade-off between inflation and deflationary pressure, as well as the unstable purchasing power make it difficult to consider bitcoin as a store of value. To conclude, bitcoin is different from traditional money because it does not fully respect the fiat currency properties, and in particular, there is no issuer responsible for it.

In fact, bitcoin is governed by a protocol run by a network of computers that are distributed around the world; government monetary policies have no direct impact on it.

In economics, there are different perspectives related to a currency. Another point of view is to see currency through institutionalist economics as a "unit of account". Based on this, bitcoin could be considered as a "currency" in the sense of money being a "social institution" (Elise Alfieri, Radu Burlacu and Geoffroy Enjolras ,2019, P.117). 


\subsection{Advantages of bitcoins over fiat money:}

(Swamy Ta, Pramanshu Shukla, Shreyas Ganesan Iyer, Rishi Gupta,2017.p.57)

1. No Regulating Authority: The first and foremost advantage of bitcoin over money exchange system is that there is no regulating authority to control the transaction as it works on peer-to-peer basis which does not need any centralized banks as they are not issued by any bank..

2. Fungibility: is the ease of exchange of one good with another,

3. Durability: It is more durable than paper currency because it cannot be destroyed as they are stored electronically.

4. Hard to counterfeit: Bitcoins are hard to counterfeit compared to money because it is not in printable format but in a password protected chain of crypto currency mathematical algorithm, which could not be accessed easily unless and until there is no hacking.

5. Reliability: The advantage is that there is no settlement risk and it is non repudiable. The savings in cost of a large settlement team

\subsection{Volatility of bitcoins:}

The Bitcoin system manifests instability from two main sources, Some economic studies have assessed market price instability to be a result of the lack of flexibility in the Bitcoin supply production. The price volatility of Bitcoin may reflect a naïve understanding by the designer of Bitcoin, in interpreting the monetary value would stabilize, by a fixed supply of Bitcoin . There is no other mechanism in place to stabilize prices. The demand for Bitcoin, regardless of the motivation for holding it, increases as the price decreases, and vice-versa. (Jolana Kubicek,2018, p.19)

There is no global regulatory framework for Bitcoin. Countries individually regulate, ignore or forbid the use of Bitcoin, and Bitcoin exchanges may or may not be regulated. Consequently, even though Bitcoin is a global currency, its price can vary around the world. Pieters and Vivanco (2017) find that Bitcoin prices are exchange and country dependent and report that Local Bitcoins P2P prices follow the same trend as exchange prices but are more volatile. (Mark Holuba, Jackie Johnsonb, 2019,P. 358)

According to research performed by Glaser et al. (2014), among others, the prices of Bitcoin are inherently determined by supply and demand. Yet, without regulative bodies, the forces of 
supply and demand may yield prices for Bitcoin that may not be economically justified or tenable. At the same time, it is expected that due to blockchain technology, working within the confines of the consensus, an increase in the number of participants in Bitcoin may increase the volume and number of participants leading to a more stable price for Bitcoin. Yet, volatility in the earlier stagemay be problematic in the short run. This hypothesis gives insight into a problem that fiat currency faces and one that governing bodies try to avoid (Ehsan Nikbakht, Manuchehr Shahrokhi, Alford Corriette,2018, P.P.5-7)

\subsection{Regulating bitcoin :}

The financial crimes enforcement network (FinCEN) directive published in 2013 reaffirmed the US Government's position that Bitcoin was not considered equal to a fiat currency. However, they did say that the Bitcoin exchange services would be treated the same as any other money service business (MSB). The difficulty was that FinCEN did not clarify what constituted an MSB for virtual currencies, especially as many bitcoin miners could be making and selling bitcoins but would not come under the category as an MSB. (Mohammed Ahmad Naheem , 2019 ,P.519)

Since this declaration in 2013 by FinCEN, there had been little progression in reaching an agreement for a regulation approach, and it was not until November 2015 that the Department of Justice held its first ever conference on the digital currency blockchain at the Federal Reserve Bank in San Francisco.This conference could be seen as finally beginning to establish common ground between the government and industry leaders in the financial technology sector, or as an admission that FinCen is still struggling to get its head around the complexities that cryptocurrencies present to the regulatory sector (Mohammed Ahmad Naheem , 2019 ,P.519)

The popularity of using bitcoins is based on the user's anonymity and the system transparency (because it uses the blockchain technology). However, participants could be reluctant to participate in this new system because bitcoin has no legal basis; companies make the choice to use bitcoin or not; the fixed costs of adopting this technology are high (sophisticated technical knowledge is needed); and there are network externalities effects (if few businesses accept bitcoin, few consumers may accept them, which in turn implies that few companies decide to accept them). 
Resolving this "vicious circle" is difficult because bitcoin is not regulated by an institution, and there is no possibility to make loans on the market. Empirical studies confirm the controversial property of "medium of exchange" based on the fact that users do not entirely turn to bitcoin for this property (Baur ,Hong , Lee , 2018,P.178).

\subsection{Adoption of Bitcoin around the world:}

Bitcoin has received a mixed welcome around the world. As has been stated in previous sections there are markets more suited to a need for Bitcoin. "Since Bitcoin is relatively new, no metric exists by which one can ascertain an exact rate of adoption, and in all likelihood, due to the complex nature of the Bitcoin network, none ever will" (Darlington, 2014, p. 4). It is worth noting that certain countries have higher rates of search volume and Bitcoin mining per capita such as Iceland (Darlington, 2014).

Two of the major countries to pass regulation on Bitcoin are China and Japan. The US is a major location of any finical news but has been slower to pass national laws on how Bitcoin will operate. A closer look at what these 3 major centers of economic power have done with Bitcoin is illustrative.

United States. The US government has so far not made any major decisions in any relation with Bitcoin, however it can look to local governments as to what results can be expected to the passage of regulatory legislation. New York was at one-point hopeful of becoming a hub of Bitcoin traffic.

However, the regulation placed on Bitcoin with a Bitlicense to hold funds for customers in an exchange has caused New York to lag behind others instead of leading the Bitcoin market (Barlyn, 2016).

China. Even more than New York and the licensing of dealing in Bitcoin China has placed a ban on all Bitcoin sales (Cheng, 2017).

In addition to just selling Bitcoins Chinese citizens are banned from trading in all cryptocurrencies in mainland China or for Chinese startups to raise money with the sale of cryptocurrencies (Rapoza, 2017).

Japan. Opposite to China's policies, Japan has embraced cryptocurrency. Whereas China has cracked down on the cryptocurrency market Japan has now readily accepted Bitcoin (Graham, 2017). 
Japan and its Finical Service Agency (FSA) already recognize 11 different companies as cryptocurrency exchange operators, Japan has in fact recognized Bitcoin as a legal tender with realtors backing the laws (Graham, 2017).

2.12 Future Acceptance of bitcoins : (Dirk G. Baur , KiHoon Hong, Adrian D. Lee, 2018, P.178 )

When evaluating the potential future use and acceptance of bitcoin it is important to analyze the growth path of bitcoin supply. The supply of bitcoins is perfectly predictable and will continue to increase in decreasing steps until 2040 and remain at the 2040 level ad infinitum. This has strong implications for the value of bitcoins and the potential deflationary effects it may entail. Since the demand for bitcoins, in contrast to its supply, is unpredictable both in the near future and beyond 2040, it is difficult to forecast the future value and usage of bitcoins.

However, if the demand increased steadily, the demand would eventually become larger than the supply leading to rising prices of bitcoin and thus deflationary effects. These built-in deflationary effects make it more likely that bitcoins will be used as an investment rather than as a medium of exchange.

If virtual currencies evolve as a medium of exchange and become an accepted alternative to fiat currencies, they will open up a new type of dual or multiple currency regime in which fiat and virtual currencies coexist. Historically, there are many examples of dual or multiple currency economies. There have been various commodity currencies as media of exchange including shells, cigarettes, cocoa beans, barley and many others. In the middle ages, gold, silver and copper coins were often circulated simultaneously at predefined exchange rates.

In the 1800s, commodity-backed monies as well as government-issued fiat currency were circulated. In the US, multiple currencies as media of exchange were common during the 1930s when privately-issued banknotes were used simultaneously with government-backed fiat and commodity-backed currency. More recently, numerous examples of dual currency economies are observed in developing and emerging economies including Liberia, Cuba and many Latin American states. Switzerland is an example of an advanced industrial country where the foreign currency euro 
is accepted in most parts despite the global acceptance of the Swiss franc.

\section{Conclusions and Future Research :}

\subsection{Conclusions :}

Cryptographic assets, including cryptocurrencies such as bitcoins have generated a significant amount of interest recently, given their rapid increase in value and volatility. As activity in bitcoins and other cryptographic assets has increased, it has attracted regulatory security across multiple jurisdictions.

bitcoin is the best-known cryptocurrency which currently holds the largest market capitalization and is regarded as a standard example of a cryptocurrency. bitcoin has emerged as the most popular virtual currency, and maintains the greatest share ahead of its competitors; the Ethereum, Ripple, Litecoin and bitcoin Cash.

bitcoin is now accepted as an alternative payment method by many merchants like Subway and Microsoft. bitcoin is available for trading in many cryptocurrency exchanges across the world. Major cryptocurrency exchanges are mostly based in US, Europe, Japan and Korea, as of November, 2017. By a variety of metrics, bitcoin is no longer a financial curiosity. From its genesis transaction of 50 bitcoins in January 2009, bitcoins in circulation now number more than 17 million. An estimated 35 million Bitcoin wallets are held worldwide with $10 \quad 0,0 \quad 0 \quad 0 \quad$ companies accepting payments in bitcoins, some via the newly issued bitcoin debit card.

Interestingly, bitcoin was released around that time as a solution to the fragile global financial system, and the academic literature draws attention to bitcoin's role as an investment shelter during stress periods such as the European debt crisis of 2010

\subsection{Future Research :}

The researcher suggests the following areas for future research in respect of this research:

1- Make more researches on the effect of accounting for bitcoins and other crypto currencies on the role of auditor while auditing financial reports.

2- Make more researches on the effect of the bitcoin price volatility on the investors .

3- Make more researches on the tax impact for bitcoins .

4-Make more researches in accounting for other cryptocurrencies which have different features than bitcoins . 


\section{Reference}

Agata, K. Paweł, M. Ida, M. Świerczyńska,K. (2019), "Bitcoin: Safe haven, hedge or diversifier? Perception of bitcoin in the context of a country's economic situation-A stochastic volatility approach" Physica A 524 (2019) 246-257.

Alfieri, E. Burlacu, E. and Enjolras, G. (2019), "On the nature and financial performance, of bitcoin", The Journal of Risk Finance Vol. 20 No. 2, 2019.PP 114-119.

Almashaqbeh, G. (2019) , "CacheCash: A Cryptocurrency-based Decentralized Content Delivery Network “,Unpublished Decorate, COLUMBIA UNIVERSITY.

Ammos, S.(2018) , "Can cryptocurrencies fulfil the functions of money?" , The Quarterly Review of Economics and Finance VOL NO. 70 .PP 4-6

Antonakakis, A. , Chatziantoniou , L. , and Gabauer, D. (2019) ," Cryptocurrency market contagion: Market uncertainty, market complexity, and dynamic portfolios", Journal of International Financial Markets, Institutions \& Money Vol No.61, P.358.

Baur ,D.G., Hong, K. and Lee, A.D.(2018)," Bitcoin: Medium of exchange or speculative assets?" , Journal of International Financial Markets, Institutions \& Money Vol No.54, P.178.

Baur, D.G., Hong, K. and Lee, A.D. (2018), "Virtual currencies: media of exchange or speculative asset?", Journal of International Financial Markets, Institutions and Money, Vol. 54, pp. 177-189.)

Easley, D. O'Hara, M. and Basu, M. ( 2019) ," From mining to markets: The evolution of bitcoin transaction fees" , Journal of Financial Economics Vol No.134.

Figuet, J.M. (2016), "Bitcoin et blockchain: quelles opportunités?", Revue d'économie financière, Vol. 123 No. 3, pp. 325338

Gronwald , M. (2019) , "Is Bitcoin a Commodity? On price jumps, demand shocks, and certainty of supply", Journal of International Money and Finance Vol No.97 pp.130139. 
Holuba, M. and Johnsonb, J. (2019), "The impact of the Bitcoin bubble of 2017 on Bitcoin's P2P market", Finance Research Letters No.29, pp 98-102.

James K. D. (2014) , "The Future of Bitcoin: Mapping the Global Adoption of World's Largest Cryptocurrency Through Benefit Analysis "University of Tennessee Honors Thesis Projects

kubicek, J.(2018), "complications of cryptocurrency : financial and cybersecurity risk in the age of bitcoin". The Journal of Risk Finance, Vol. 18 Issue: 2, pp.125-139.

Naheem , M.A.(2019) , "Exploring the links between AML, digital currencies and blockchain technology" , Journal of Money Laundering Control Vol. 22 No. 3, 2019 .pp 517-519.

Nikbakht, E. Shahrokhi, M. and Corriette, A. (2018) ," Blockchain \& distributed financial data", www.emeraldinsight.com/0307-4358.htm

Philippasa, D. Rjibaa, H. Guesmib, K. and Gouttec, S. (2019), "Media attention and Bitcoin prices", Finance Research Letters .

Swamy, T, Shukla, P. , Ganesan , L. and, Gupta,R. (2017) "Review Paper on Emergence of Bitcoin in India, its Technological Aspects and Legal Implications , VIT Business School, VIT University, Vellore - 632014| swamy.t@vit.ac.in, P.57.

Ziyu, W., Hui, Y., Zongyang, Z. Piao, J. and Liu, J. (2020), "ECDSA weak randomness in Bitcoin " , Future Generation Computer Systems No.102 . 